\title{
Responses of Thin Film Photovoltaic Cell to Irradiation under Double Laser Beams of Different Wavelength
}

\author{
Yuchen Yuan ${ }^{1,2, a}$, Chenwu Wu ${ }^{1, b^{*}}$, Guangnan Chen ${ }^{1, c}$ \\ ${ }^{1}$ Institute of Mechanics, Chinese Academy of Sciences, No.15 Beisihuanxi Road, Beijing 100190, \\ China \\ ${ }^{2}$ Graduate University of Chinese Academy of Sciences, No.80 Zhongguancun East Road, Beijing \\ 100080, China \\ a yuanyuchen11@mails.gucas.ac.cn, ${ }^{\text {b* }}$ chenwuwu@imech.ac.cn, ${ }^{c}$ gnchen@imech.ac.cn
}

Keywords: Laser, Thin-film photovoltaic cell, Heat, Temperature elevation, Efficiency.

\begin{abstract}
During the process of Laser beam long-range energy transmission based on the photovoltaic principle, the temperature elevation of the photovoltaic cell under Laser irradiation would greatly affect its photoelectric conversion efficiency. In this work, the heat generation mechanism of thin-film photovoltaic cell under Laser irradiation was analyzed based on the energy conversion relationship between light, electricity and heat. The output voltage characteristic of a thin film $\mathrm{GaInP} / \mathrm{GaAs} / \mathrm{Ge}$ triple-junction photovoltaic cell under irradiation of dual-wavelength Laser beam was investigated, and the results show the dependence of the conversion efficiency on the temperature and the valve effect, which was caused by the long wavelength Laser and would sharply reduce the photovoltaic conversion efficiency of short wavelength Laser. The temperature field of the multi-layer structure subjected to Laser irradiation was analyzed by finite element method, and the results indicate that the temperature elevation pattern depends on the spatial distribution of the light absorption and can be adjusted via optimizing the photovoltaic cell structure.
\end{abstract}

\section{Introduction}

Long-range energy transmission may perfectly solve the problems of energy supplying of the Unmanned Aerial Vehicle (UAV) and Solar Power Station (SPS). It could greatly prolong the UAV's duration of flight and therefore reduce the frequency of landing and refuel [1]. On the other hand, considering the huge cost for the traditional energy transmission methods of Solar Power Station, the advantages of long-range energy transmission are more prominent [2].

Up to now, the long-range energy transmission might be realized either by microwave or Laser beam. Compared with microwave, the system based on Laser beam can be established by a smaller emitter/receiver, and the energy loss is lower [3]. The Laser based long-range energy transmission mainly includes two opposite converting processes between electric energy and light energy, in which the solar cell is used to change the light energy of Laser beam into electric energy. In particular, III-V multi-layer thin-film solar cell has been widely used in these situations due to its superiority in light weight, high conversion efficiency and good resistance to radiation degradation, etc. [4, 5]. Actually, the thin-film solar cell is always an ideal choice for powering the UAVs and SPS, of which the comprehensive performance is very sensitive to its self-weight.

The efficiency of the Laser based energy transmission surely has a strong dependence on the photovoltaic conversion efficiency. Furthermore, during the photovoltaic conversion process, the temperature of the solar cell would be rather high due to the relatively high energy density of the Laser beam, and thus would lead to a significantly reduction in its efficiency. Generally speaking, the relationship between the open-circuit voltage of the solar cell and its temperature $[6,7]$ means that the open-circuit voltage decreases when temperature rises, as the temperature elevation would also reduce the fill factor and further reduce the overall efficiency of solar cell. Moreover, as aforementioned, the energy flux of Laser beam is very great- just like much highly concentrated sun light-and the energy of light couldn't be converted to electric energy completely. Much of the light energy would be dissipated as heat energy and thus brought a higher temperature to the solar cell. These entirely mean that the efficiency loss caused by the temperature elevation may be the dominant 
factor influencing the performance of Laser based long range energy transmission. Therefore, we need to investigate the temperature elevation of the solar cell under Laser irradiation and its effect on the cell efficiency to optimize the design of energy transmission systems.

In this paper, the concept of irradiating the triple junction thin-film solar cell with two Laser beam of different wavelength covering the two absorption peaks of the cell was described. The heat generation and temperature elevation of the solar cell and its effect on the efficiency were studied experimentally. Finally, the mechanism of heat generation in the process of energy conversion in solar cell was analyzed and the finite element model was established to investigate the temperature field of solar cell under typical condition. The methodology of this research could be used for further optimistic design of Laser based long range energy transmission.

\section{Experiment and results}

Fig. 1(a) shows the experimental platform including Laser, measurement device and tested cells. The structure sketch of tested cells-GaInP/GaAs/Ge triple-junction photovoltaic cell is shown in Fig. 1(b). Considering the light absorption characteristic of the cell, we choose two different Laser beams-one's wavelength is $532 \mathrm{~nm}(30 \mathrm{~mW})$ and the other's wavelength is $1064 \mathrm{~nm}$ (about $25 \mathrm{~W}$ ), which correspond to the absorption peaks of the GaInP layer and Ge layer, respectively.

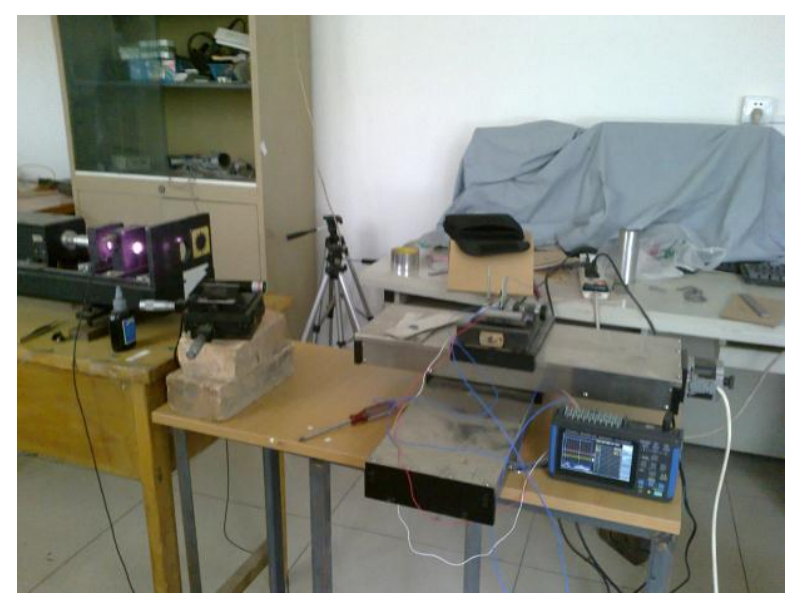

(a)

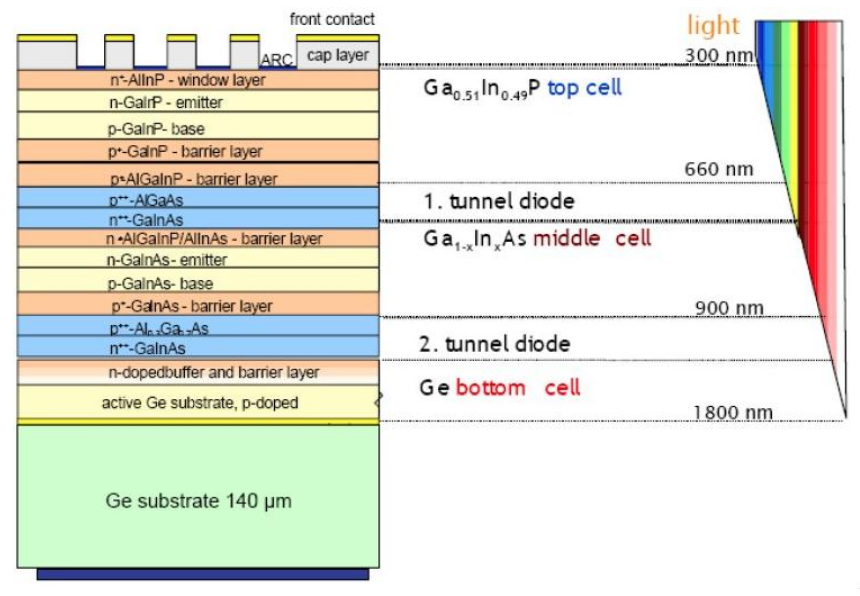

(b)

Fig. 1(a) experimental platform (b) sketch of the solar cell [8]

The thermal imager shown in Fig. 2(a) has been used to record the temperature field of the solar cell and the Al basement, and the adjustable clamp has been used to hold the cell and make sure that the two Laser spot on the cell surface are concentric.

At the beginning of the experiment, the cell is under irradiation of the short wavelength Laser beam $(532 \mathrm{~nm}, 30 \mathrm{~mW})$. Then the long wavelength Laser beam $(1064 \mathrm{~nm}$, about $25 \mathrm{~W})$ is introduced to irradiate the cell simultaneously. After a period of time, the long wavelength Laser beam is removed to leave the solar cell being irritated only by the short wavelength Laser beam. Then the solar cell would be gradually cooled by air convection. The output voltage and the temperature of the cell are recorded all along. Therefore, the temperature elevation as well as the dependency of solar cell efficiency on temperature could be analyzed. As a result, Fig. 2(b) shows the typical output voltage curve of the cell under a round of temperature rising and dropping.

As shown in Fig. 2(b), wherein the numbers marked next to the thermal image refer to the maximum temperature, the irradiation of long wavelength Laser beam would lead to obvious increasing in output voltage right after it is introduced, which results in the small sharp peak in the curve. But the voltage output declines rapidly after that peak, which should be due to the quick temperature rising of the cell. In detail, the output voltage almost reduced to 0 when the peak temperature reaches over $250^{\circ} \mathrm{C}$. This reveals that the conversion efficiency of the solar cell strongly depends on its temperature. It also seems like that the long wavelength Laser produces a valve effect on the response of the cell to the short wavelength Laser, i.e. the thermal effect of the long wavelength 
Laser finally cut out the conversion of the short wavelength Laser energy. After the long wavelength Laser beam is removed and the cell is cooled, the output voltage gradually restores and finally reaches the initial level. This also indicates that the effect of temporary temperature rising on the photovoltaic cell is non-permanent to some extent. Above all, the results show that such double Laser beam irradiation would improve the total efficiency of the multi-junction photovoltaic cell if the temperature elevation can be controlled, which requires for optimization of the energy distribution of different Laser beams to avoid the inappropriate heat accumulation.

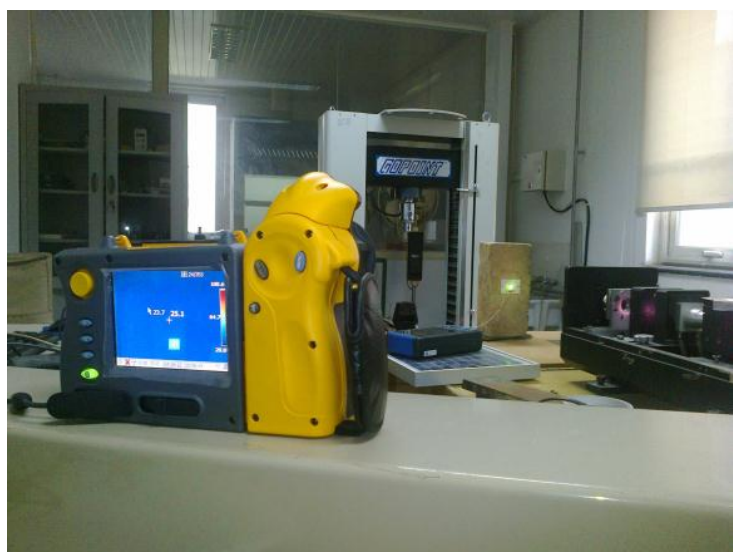

(a)

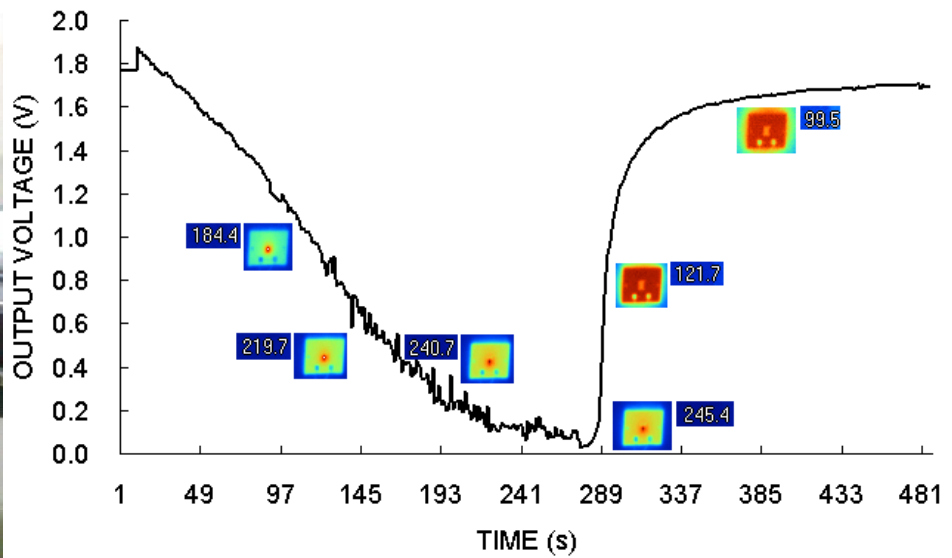

(b)

Fig. 2(a) Thermal image device (b) The output voltage and cell temperature field

\section{Theoretical analysis and discussion}

The multi-layer structure of the solar cells could be simply treated as three layers: GaInP layer, GaAs layer and Ge layer. The study is based on the commercial finite element program and the heat transfer numerical model of the solar cell is established. The finite element method and the time differencing method is used to solve the multi-layer structure nonlinear Fourier heat transfer equation:

$$
\frac{\partial}{\partial x}\left(k(T) \frac{\partial T}{\partial x}\right)+\frac{\partial}{\partial y}\left(k(T) \frac{\partial T}{\partial y}\right)+\frac{\partial}{\partial z}\left(k(T) \frac{\partial T}{\partial z}\right)+q_{v}=\rho c \frac{\partial T}{\partial t}
$$

Where $x / y / z$ axis is the orthogonal axis, $T$ is the temperature, $t$ is the time variable, $\rho$ is the material density, $c$ is the specific heat, $k$ is the thermal conductivity, and $q_{v}$ represents the heat generation rate per unit volume, for which the absorbed Laser energy is distributed as follows: GaInP layer 0.067W, GaAs layer $0.35 \mathrm{~W}$, Ge layer $24.58 \mathrm{~W}$ according to the basic absorption law [9].

In addition, taking into account the symmetry of the cell and Al basement, only $1 / 4$ model is taken to be calculated. The entire surface of the cell is divided into $20 \times 20$ units, and each layer is divided into 5 units in the thickness direction of the cell. The Al basement is only sparse meshed, and the thickness direction of the basement is meshed into 10 units. The whole structure and the 1/4 symmetric finite element model is shown in Fig. 3. The Laser irradiation is simply treated as an internal heat generation rate load, and the system heat mainly dissipates by air convective heat transfer.

The solar cell used in experiment is multi-layer structure, and is simplified into 4 -layers structure (including the Ge substrate layer). The structure of the model is: GaInP layer $(7 \mu \mathrm{m}) / \mathrm{GaAs}$ Layer $(15 \mu \mathrm{m}) /$ Ge layer $(13 \mu \mathrm{m}) / \mathrm{Ge}$ substrate layer $(140 \mu \mathrm{m})$. The temperature and the normal heat flux at every interface meet the continuity condition.

The cell is adhered to the Al basement, so in the model there is a layer of low thermal conductivity material which represents the thermal contact resistance between the Ge substrate and Al basement. The temperature and the normal heat flux of the connecting interface between this thin layer and the solar cell/ Al basement also meet the continuity condition. 
The cylindrical internal heat generation rate load is applied to the solar cell, and the thermal convection boundary condition is applied to the surface of the cell and Al basement. The diameter of the Laser spot is $2 \mathrm{~mm}$. Considering that the energy of the Laser obey the Gaussian distribution, the cylindrical radius of the heat generation rate load is $1.5 \mathrm{~mm}$. For simplicity, the absorption of the Laser energy is assumed to be uniform in the thickness direction of each layer of the cell. The testing environment is: room temperature, natural convection of air; the room temperature is $25{ }^{\circ} \mathrm{C}$, and the convection coefficient is set to 10 [10].

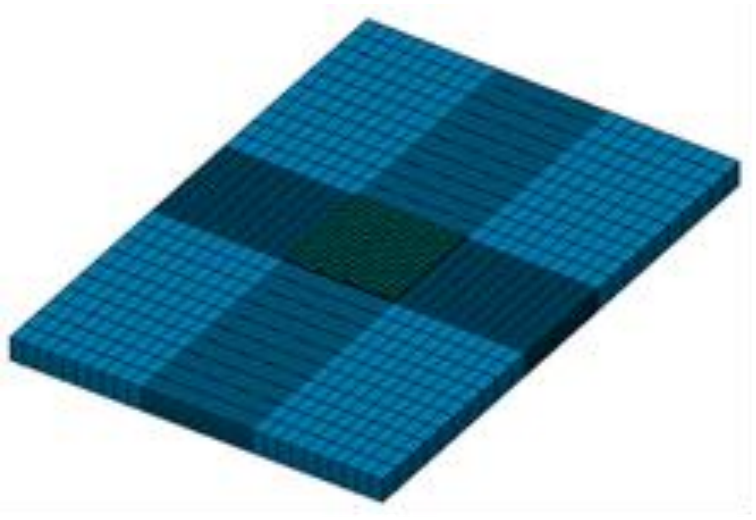

(a)

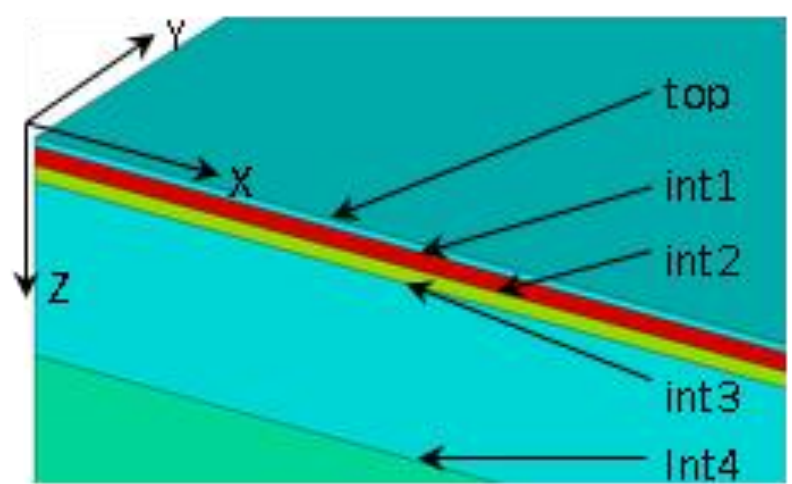

(b)

Fig. 3 (a) finite element discrete model of the whole structure (b) the 1/4 symmetric model

During the experiment, the duration of long wavelength Laser irradiation is 170s, and after the long wavelength Laser beam is removed, the cooling time is about 220s; in order to simulate the whole experimental process, the total length of time of the finite element calculation is set to 390s. During the first 170s of time, the internal heat generation rate load is applied and the heat generation is calculated, and in the next 220s the load is removed and the cooling process is calculated. As a preliminary analysis, a typical calculation result is obtained. The calculation result of temperature field and the total heat flux field at 170s moment is shown in Fig. 4 and 5. Fig. 4(a) shows the temperature field of the model, and Fig. 4(b) shows the total heat flux field; Fig. 5(a) shows the distribution of temperature along the $\mathrm{x}$ axis of the interface/surface, and Fig. 5(b) shows the total heat flux along the same direction. It is worth mentioning that although the computational model is a $1 / 4$ symmetric model, but the contour given in Fig. 4 has been symmetry expanded, so the figures show the results of the whole model. According to the result shown in Fig. 4, the highest value of temperature (about $250{ }^{\circ} \mathrm{C}$ ) appears at the central region of the cell which is under Laser irradiation, and the highest value of total heat flux which is appeared at the edge of the region shows that there is a peak value of temperature gradient. Also as the Laser energy is highly concentrated and the thermal conductivity of semi-conductor material is generally small, there are big temperature gradients either along the $\mathrm{x}$ axis and thickness direction.

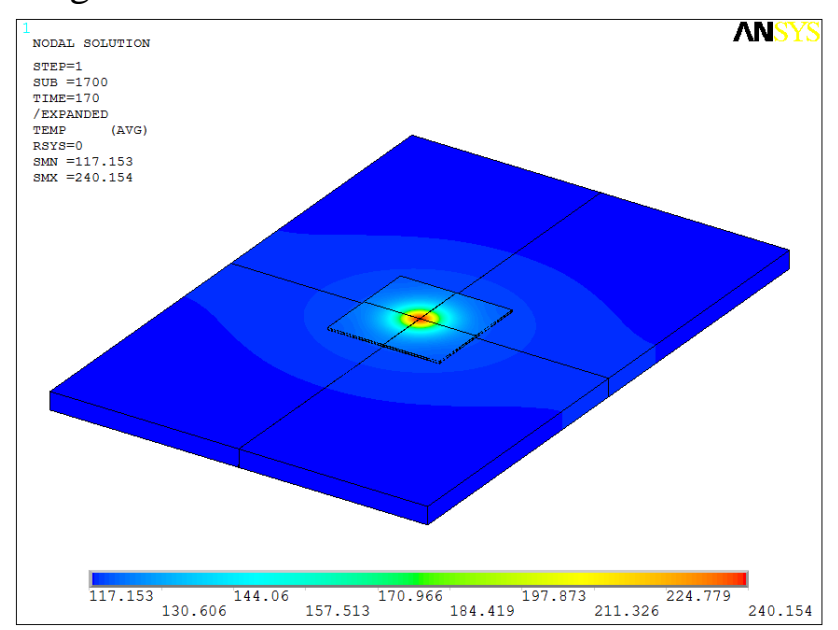

(a)

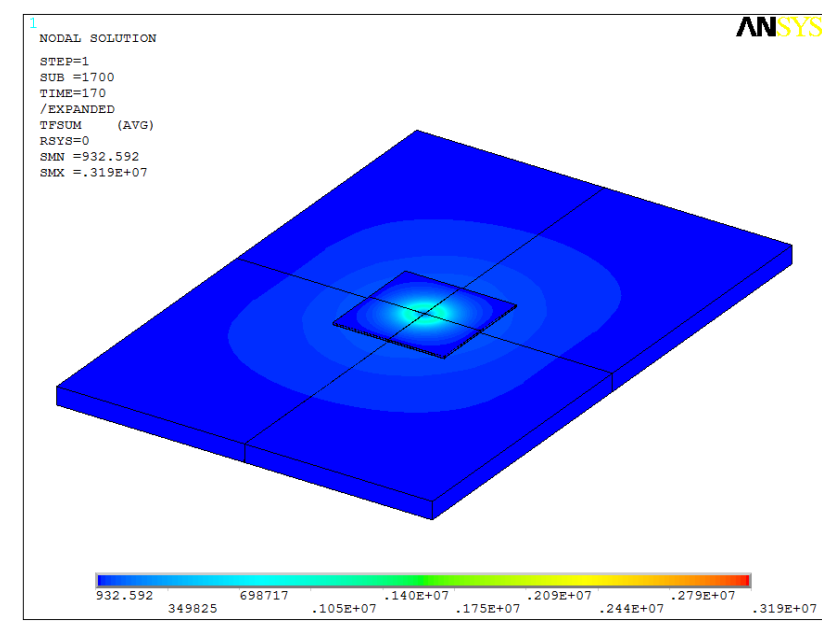

(b)

Fig. 4 (a) temperature field and (b) total heat flux field at 170s moment 


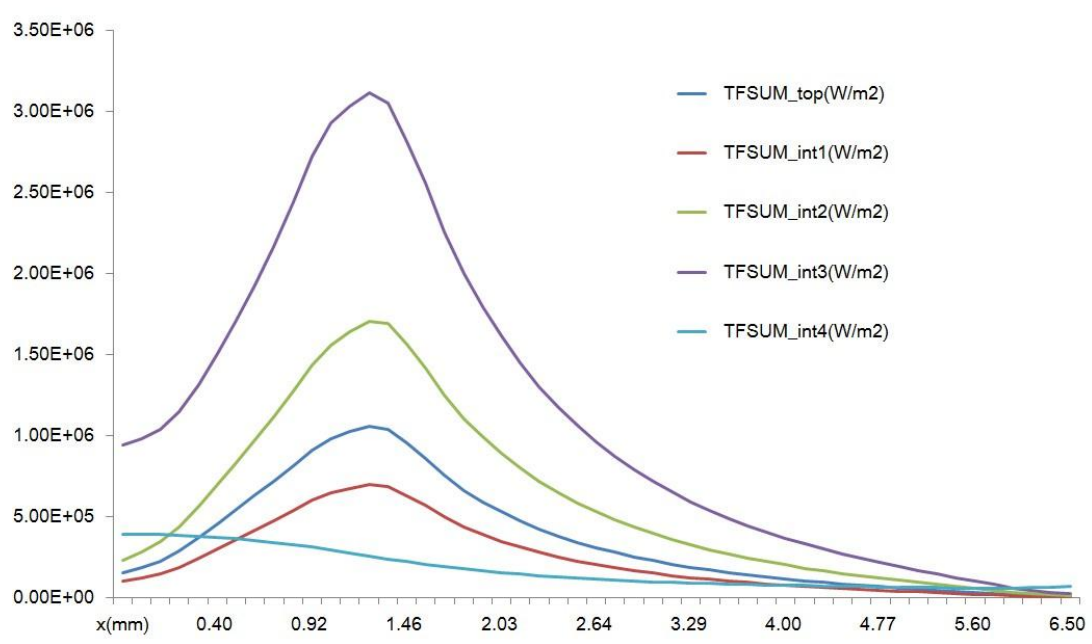

(a)

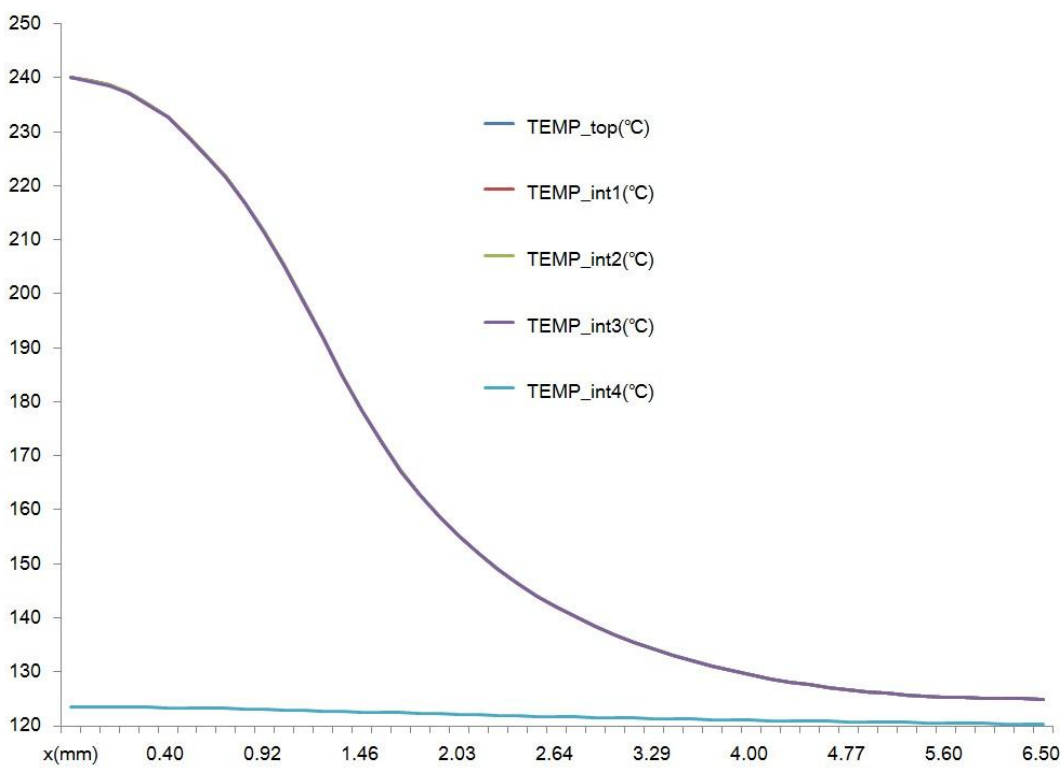

(b)

Fig. 5 (a) The temperature and (b) the total heat flux of surface/interface along the $\mathrm{x}$ axis

In Fig. 5, the subscript "_top" represents the path on the surface of the cell, “_int1", “_int2", "_int3" and "_int4" represent the path on the interface of GaInP/GaAs, GaAs/Ge, Ge/Ge substrate, and Ge substrate/Al basement, respectively. According to the result of calculation, the temperature distribution along $\mathrm{x}$ axis on the surface and internal interface of the cell is very similar. It shows that the thermal resistance of the interface is very small in the thickness direction of the cell. As the thermal resistance caused by the assembling process of the cell and Al basement is comparatively high, so the heat couldn't transfer from the cell to the aluminum plate easily and there is a significant difference between the temperature distribution curve of cell and $\mathrm{Al}$ basement.

A ring-shape distribution of the highest value of the total heat flux appears near the Laser irradiation point. Because the energy of Laser beam is highly concentrated, the temperature distribution at the region under Laser irradiation is almost even and therefore no large heat flux appears at this region. The energy input by the Laser would be transferred to other region by thermal conduction, so the highest value of total heat flux appears at the edge of the Laser irradiation region. Fig. 5(b) shows the differences of heat flux in each interface due to the differences between the thermal conductivity of each material. Furthermore, as the thermal conductivity of Al basement is relatively high, so the temperature distribution in the basement is more even and the total heat flux in it is far smaller than in the cell. The result could be a preliminary reference for the follow-up cell thermal design and some optimization and calculation methods in this paper could be used in the design. 


\section{Conclusion}

The concept of irradiating solar cells by dual-wavelength Laser beam to realize the long-range energy transmission is described.

1.The long wavelength Laser could increase the output voltage of the cell at the beginning of irradiation, while the total output voltage would decrease when the power of long wavelength Laser reaches a certain value.

2. The thermal generation model of the solar cell and the heat conduction model have been established and the governing equation is solved by using finite element method and time differencing method.

3. The temperature elevation pattern depends on the spatial distribution of the light absorption and can be adjusted via optimizing the photovoltaic cell structure.

4.The model provides a theoretical prediction of the temperature elevation of solar cell under dual-Laser beam irradiation, which could be used in the future optimistic design of the long-range energy transmission system.

\section{Acknowledgements}

This work was supported by the National Natural Science Foundation of China (Grant No. 11002145).

\section{References}

[1] Information on http://lasermotive.com/

[2] F.D. M., What Is a Solar Power Satellite? in: J.N. Pelton (Eds.), Solar Power Satellites, Springer New York, 2012, pp. 1-8.

[3] P.E. Glaser, Power from the sun: its future, Science 162 (1968) 857-861.

[4] M.A. Green, K. Emery, Y. Hishikawa, W. Warta, Solar cell efficiency tables (version 37), Progress in Photovoltaics: Research and Applications 19 (2011) 84-92.

[5] R.R. King, D.C. Law, K.M. Edmondson, et al., 40\% efficient metamorphic GaInP/GaInAs/Ge multi-junction solar cells, Appl. Phys. Lett., 90 (2007) 183516.

[6] S.M. Sze, K.K. Ng, Physics of Semiconductor Devices, third ed., John Wiley \& Sons, New Jersey, 1990.

[7] S.Z. Xiong, M.F. Zhu, The Fundaments and Applications of Photovoltaic cells, Science Press, Beijing, 2009.

[8] Information on http://www.ys-solar.com/

[9] Information on http://refractiveindex.info/

[10] J.H. Lienhard IV, J.H. Lienhard V, A heat transfer textbook, third ed., Phlogiston Press, Cambridge, Massachusetts, 2005. 
Energy and Environment Materials

10.4028/www.scientific.net/MSF.743-744

Responses of Thin Film Photovoltaic Cell to Irradiation under Double Laser Beams of Different Wavelength

10.4028/www.scientific.net/MSF.743-744.937 